\title{
Development of single mouse blastomeres into blastocysts, outgrowths and the establishment of embryonic stem cells
}

\author{
Chanchao Lorthongpanich ${ }^{1,4}$, Shang-Hsun Yang ${ }^{1,2,3}$, Karolina Piotrowska-Nitsche ${ }^{1,2}$, Rangsun Parnpai ${ }^{4}$ \\ and Anthony W S Chan ${ }^{1,2,3}$
}

\begin{abstract}
${ }^{1}$ Yerkes National Primate Research Center, ${ }^{2}$ Department of Human Genetics and ${ }^{3}$ Genetics and Molecular Biology Program, Emory University School of Medicine, Suite 2212, 954 Gatewood Road, North East Atlanta, Georgia 30329, USA, ${ }^{4}$ Embryo Technology and Stem Cell Research Center, Suranaree University of Technology, Nakhon Ratchasima 30000, Thailand
\end{abstract}

Correspondence should be addressed to R Parnpai; Email: rangsun@sut.ac.th

A W S Chan; Email: achan@genetics.emory.edu

Reproduction (2008) 136275

The authors and journal apologize for an error in the above paper, which appeared in volume 135 part 6, page 805. The author list and affiliations should read as follows:

Chanchao Lorthongpanich ${ }^{1,4}$, Shang-Hsun Yang ${ }^{1,2,3}$, Karolina Piotrowska-Nitsche ${ }^{1,2,5}$, Rangsun Parnpai ${ }^{4}$ and Anthony W S Chan ${ }^{1,2,3}$

\footnotetext{
${ }^{1}$ Yerkes National Primate Research Center, ${ }^{2}$ Department of Human Genetics and ${ }^{3}$ Genetics and Molecular Biology Program, Emory University School of Medicine, Suite 2212, 954 Gatewood Road, North East Atlanta, Georgia 30329, USA, ${ }^{4}$ Embryo Technology and Stem Cell Research Center, Suranaree University of Technology, Nakhon Ratchasima 30000, Thailand and ${ }^{5}$ Department of Experimental Embryology, Institute of Genetics and Animal Breeding, Polish Academy of Sciences, 05-552 Wolka-Kosowska, Poland
} 